\title{
Challenges Faced by an Otolaryngology Resident During Covid-19 Pandemic: An Indian Perspective
}

\author{
Raghul Sekar $^{1} \cdot$ Arun Alexander $^{1}$ (D) Sivaraman Ganesan $^{1}$
}

Received: 5 August 2020/Accepted: 24 August 2020/Published online: 1 September 2020

(C) Association of Otolaryngologists of India 2020

\begin{abstract}
SARS-CoV-2 virus causing COVID-19 disease has brought tremendous strain on health care sectors around the world. Being a respiratory disease, COVID-19 has tremendous implications on the otolaryngology residency. Otolaryngology residents are dealing with great apprehension of getting infected while working with both COVID-19 and non-COVID patients. Further, drastic change in the routine has affected their residency training. Outpatient and Inpatient care, elective and emergency surgeries which are a crucial part of the residency training have taken a back seat. India being a densely populated country is still in the midst of the pandemic with cases increasing exponentially each day. This article enumerates the challenges faced by an otolaryngology resident during COVID-19 pandemic in a tertiary care hospital in India.
\end{abstract}

Keywords COVID-19 $\cdot$ Otolaryngology $\cdot$ Residency

\section{Introduction}

The world is under the grip of the SARS-Cov-2 virus which causes the novel corona virus disease (COVID-19). This disease was declared a pandemic by the WHO on March 11, 2020 [1]. Around 213 countries have been reported to be affected by this disease. India, being a densely populated country, is one of the worst affected in the world with over half a million cases by the end of June 2020 [2]. This

Arun Alexander

arunalexandercmc@gmail.com

1 Department of Otorhinolaryngology, Jawaharlal Institute of Postgraduate Medical Education and Research, Pondicherry 605005, India has brought unprecedented changes in health care system around the world. There is a paradigm shift in the functioning of health care: with the health system struggling to balance the care delivered to COVID and Non-COVID patients.

Residents and fellows form the major group of frontline workers along with nurses in tackling this pandemic. They shuffle between working with COVID positive patients and returning to treat non-COVID patients in their respective department following a period of quarantine. This has brought a tremendous strain on their residency training which normally lasts only for a finite period. If the epidemiologic projections hold true, this pandemic may outlast numerous residents' training programs.

COVID-19 being primarily a respiratory disease, has tremendous implications in otolaryngology training and patient care. While respiratory droplet is the major mode of transmission, there are reports of feco-oral transmission and through the conjunctiva [3]. The symptoms similar to influenza and other upper respiratory infections include fever, cough, headache, sore throat, nasal congestion, and anosmia [4]. Patients with these symptoms are commonly treated by Otolaryngologists. Thus, every patient seen in the out-patient clinic is approached with great apprehension. This paper aims to discusses the challenges faced by an Otolaryngology resident in the midst of the pandemic in India.

\section{Out-Patient and Endoscopy Unit}

Teaching hospitals have the unique responsibility of balancing the delivery of health care and the training of residents [5]. With the pandemic spreading widely, governments have brought strict lockdown measures and 
orders to cut down the workforce to curb the movement of people and restrict the spread of the virus. This has led to a drastic reduction in the outpatient visits by the patients. Telephonic consultations have been initiated by hospitals to restrict the influx of patients to the hospital and prevent overcrowding. Patient visit in ENT are restricted to head and neck malignancies and emergencies. In a busy tertiary care centre like ours, between 250 and 300 patients were seen in the outpatient department each day prior to the pandemic. This number has fallen to $15-20$ patients a day in the last three months. (Fig. 1) Many diagnoses in ENT are made clinically by Otoscopy, anterior rhinoscopy and indirect laryngoscopy which lead to aerosol generation and are avoided where possible. The use of PPE such as N95 respirators and Face shields make examination difficult. These factors have affected the skill of clinical examination especially among residents.

Prior to the onset of the pandemic 40-50 endoscopies were performed each day by residents in the endoscopy facility attached to our outpatient clinic. This being a fundamental skill in Otolaryngology residency, has been indefinitely stopped during the pandemic. Though many countries in the western world have started doing endoscopies with PPE including full body coveralls, the resource constrains in a country like India have led to the suspension of endoscopy services in the out-patient clinic.

\section{Ward/In Patient Unit}

Due to rising number of COVID patients, workforce in our hospital required reorganisation. Many general wards have been closed to mobilise health care workers to manage COVID positive patients in a dedicated ward. Ventilators and ICU beds in ENT and many surgical departments were also shifted for the care of COVID patients. Similarly, residents were divided into teams with only one team working at a time, while the other kept as backup/ quarantine. The number of patients has fallen to less than $25 \%$ of the pre-pandemic numbers and most cases admitted are patients requiring emergency care only. (Fig. 2) Elaborate ward rounds with consultants teaching and testing residents' knowledge bedside has been greatly curtailed. This has a bearing on the training of the residents.

\section{Elective Surgeries}

Surgical procedures in otolaryngology are associated with high risk of virus transmission due to the following reasons

a. Upper aerodigestive tract in the point of entry, exit and nidus for novel corona virus.

b. Upper aerodigestive tract procedures are associated with high aerosol generation.

c. Instrumentation during the procedure such as microdebrider and drill usage during these procedures are associated with prolonged aerosolization [6].

These reasons place otolaryngologists at a high risk for contacting COVID-19. Furthermore, the risk to patients of pulmonary complications and mortality in the postoperative period is high in the event of a perioperative SARSCoV-2 infection [7]. As per government guidelines, our elective operation theatres (OTs) have been shut to reduce risk of disease transmission and the redeployment of theatre personnel to the dedicated COVID facility. Surgeries such as adenotonsillectomy, septoplasty, tympanoplasty, mastoidectomy and Functional endoscopic sinus surgery are commonly the surgeries done by residents during their training. These elective surgeries have been stopped at the present times. Except cadaveric temporal bone dissection, residents cannot learn any of the above procedures outside the OT. ENT as a specialty utilizes numerous powered instruments which requires assisting senior surgeons and performing surgery under supervision to learn how to use them. This has taken a back seat in resident training.
Fig. 1 Outpatient services in 2020 and 2019 showing significant fall in outpatient cases
OUTPATIENT SERVICES

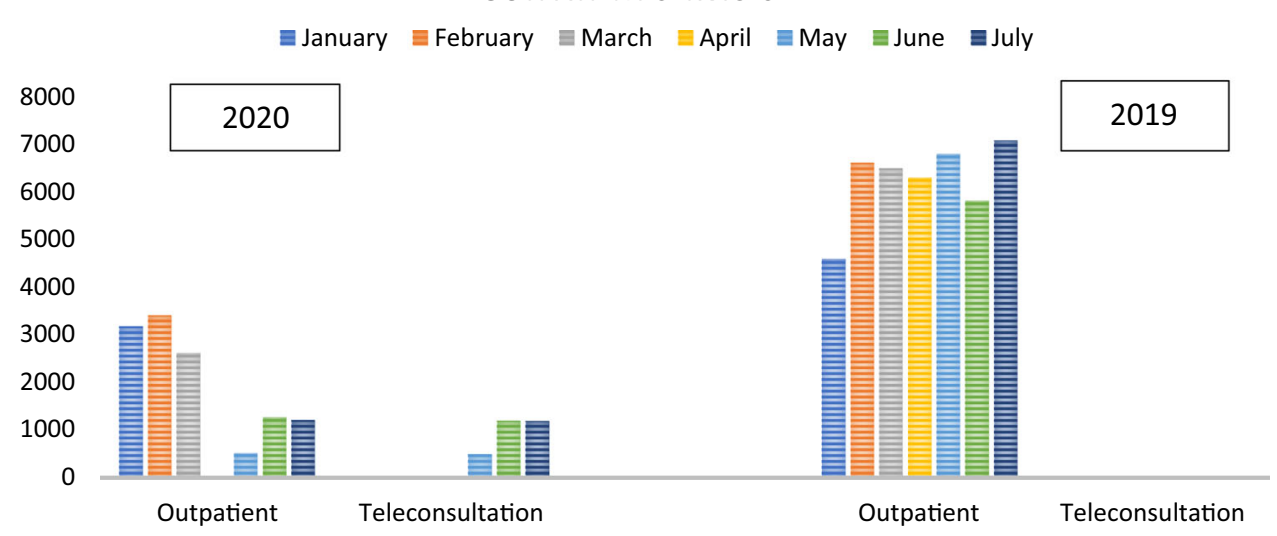


Fig. 2 Inpatient services in2020 and 2019 showing significant reduction in inpatient admissions and elective surgery. Emergency surgery remained largely unaffected

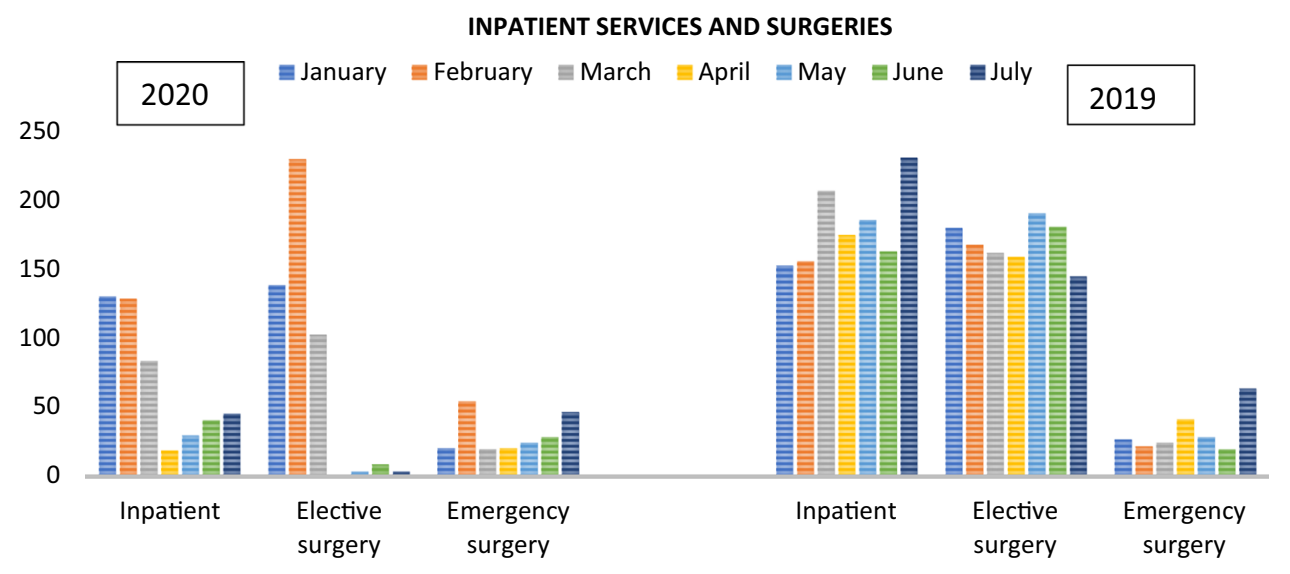

Elective surgeries in our hospital are restricted to Tier 3a and $3 b$ cases [8] with only one OT for Otolaryngology per week. Only head and neck malignancy surgeries which are presently being done and that to by senior consultants so as to reduce the duration of surgeries, exposure to aerosols and complications. This has caused substantial reduction in residents' daily surgical activity such as preoperative work up, postoperative care and involvement in surgical theatre.

\section{Emergency Surgeries}

Otolaryngology has numerous emergencies related to the upper aerodigestive tract. These require immediate work up and surgical intervention. Some of the common emergency procedures include emergency tracheostomy, tracheabronchoscopy and esophagoscopy for foreign body removal, nasal packing, peritonsillar/retropharyngeal abscess drainage are associated with high occupational risk to health care workers. Over the last 2 months there has been gradual increase in the number of patients presenting to our emergency as most of the smaller hospitals in the have stopped admitting patients. Presently, all the patients requiring emergency intervention are assumed to be COVID positive and intervention is initiated without waiting for RT-PCR report in those patients who cannot wait for the results of the throat swab. This means the surgeon and the assistant has to wear level 3 PPE (surgical cap, goggles, coverall, N95 respirator, gloves, shoe cap) during the procedure. Operating under such conditions require certain level of skill and experience aimed at reducing complications and aerosolization during the procedure. Hence these procedures are performed by senior residents/consultants. This again impairs the developing surgical skills of the junior residents.

\section{Academic Activities}

Due to the advent of latest technology and availability of smart learning tools, academic activities continue to happen unhindered by the pandemic. Conferences and CMEs have been temporarily suspended impairing resident interactions with consultants around the country and scientific paper presentations.

\section{Conclusions}

Otolaryngology residents have a high risk of contracting the disease during the continuing pandemic that has no sight in end. They have to take care of COVID positive as well as non-COVID patients and work in a stressful environment with a constant fear of getting infected during the process. At the same time, they are acutely aware of the need to obtain the academic and surgical skills needed before the end of their training. Adapting to these changing times and balancing between patient care and training is undoubtedly a difficult proposition. Hopefully, we will see the light at the end of the tunnel soon!

\section{Funding None.}

\section{Compliance with Ethical Standards}

Conflict of interest The authors declare that they have no conflict of interest.

\section{References}

1. World Health Organization. Coronavirus disease (COVID-19) pandemic.

www.who.int/emergencies/diseases/novel-coronavirus-2019

2. Worldometers.info (2020) Coronavirus update (live): 10,082,613 cases and 501,308 deaths from COVID-19 virus pandemic- 
worldometer.

https://www.worldometers.info/coronavirus/. Accessed 28 June 2020

3. Li JO, Lam DSC, Chen Y, Ting DSW (2020) Novel coronavirus disease 2019 (COVID-19): the importance of recognising possible early ocular manifestation and using protective eyewear. $\mathrm{Br} \mathrm{J}$ Ophthalmol 104(3):297-298. https://doi.org/10.1136/bjophthalmol-2020-315994

4. Guan WJ, Ni ZY, Hu Y et al (2019) Clinical characteristics of coronavirus disease 2019 in China. N Engl J Med. https://doi.org/10.1056/NEJMoa2002032

5. Pugno PA, Gillanders WR, Kozakowski SM (2010) The direct, indirect, and intangible benefits of graduate medical education programs to their sponsoring institutions and communities. J Grad
Med Educ 2(2):154-159. https://doi.org/10.4300/JGME-D-09-00 008.1

6. Mohfw.gov.in (2020) https://www.mohfw.gov.in/pdf/ENTCOVI D0306.pdf. Accessed 28 June 2020

7. COVIDSurg Collaborative (2020) Mortality and pulmonary complications in patients undergoing surgery with perioperative SARS-CoV-2 infection: an international cohort study. Lancet. https://doi.org/10.1016/s0140-6736(20)31182-x

8. Cms.gov (2020) https://www.cms.gov/files/document/covid-electi ve-surgery-recommendations.pdf. Accessed 28 June 2020

Publisher's Note Springer Nature remains neutral with regard to jurisdictional claims in published maps and institutional affiliations. 